\title{
Characterization and application of two RANK-specific antibodies with different biological activities
}

Mélanie Chypre ${ }^{1,2}$, Jonathan Seaman ${ }^{3}$, Olga G. Cordeiro ${ }^{1}$, Laure Willen ${ }^{4}$, Kathryn A. Knoop ${ }^{5}$, Andrew Buchanan $^{3}$, Richard C.A. Sainson ${ }^{3}$, Ifor R. Williams ${ }^{5}$, Hideo Yagita ${ }^{6}$, Pascal Schneider ${ }^{4}$, Christopher G. Mueller $^{1,7}$

${ }^{1}$ CNRS UPR 3572, Laboratory of Immunopathology and Therapeutic Chemistry, University of Strasbourg, 67000 Strasbourg, France

${ }^{2}$ Prestwick Chemical, Blvd Gonthier d'Andernach, Parc d'innovation, 67400 Illkirch, France

${ }^{3}$ Medlmmune, Granta Park, Cambridge CB21 6GH, UK

${ }^{4}$ Department of Biochemistry, University of Lausanne, Epalinges, Switzerland

${ }^{5}$ Department of Pathology and Laboratory Medicine, Emory University School of Medicine, Atlanta, GA 30322, USA

${ }^{6}$ Department of Immunology, Juntendo University School of Medicine, Tokyo 113-8421, Japan

${ }^{7}$ Corresponding author: Tel: +33 (0)3 884171 14, Fax. +33 (0)3 88610680

c.mueller@ibmc-cnrs.unistra.fr

Abbreviations: RANK, Receptor Activator of NF- $\mathrm{BB}$; OPG, osteoprotegerin; scFv, single-chain variable fragment; Microfold cells, M cells 


\begin{abstract}
Antibodies play an important role in therapy and investigative biomedical research. The TNF-family member Receptor Activator of NF-KB (RANK) is known for its role in bone homeostasis and is increasingly recognized as a central player in immune regulation and epithelial cell activation. However, the study of RANK biology has been hampered by missing or insufficient characterization of high affinity tools that recognize RANK. Here, we present a careful description and comparison of two antibodies, RANK-02 obtained by phage display (Newa, 2014 [1]) and R12-31 generated by immunization (Kamijo 2006 [2]). We found that both antibodies recognized mouse RANK with high affinity, while RANK-02 and R12-31 recognized human RANK with high and lower affinities, respectively. Using a cell apoptosis assay based on stimulation of a RANK:Fas fusion protein, and a cellular NF-KB signaling assay, we showed that R12-31 was agonist for both species. R12-31 interfered little or not at all with the binding of RANKL to RANK, in contrast to RANK-02 that efficiently prevented this interaction. Depending on the assay and species, RANK-02 was either a weak agonist or a partial antagonist of RANK. Both antibodies recognized human Langerhans cells, previously shown to express RANK, while dermal dendritic cells were poorly labeled. In vivo R12-31 agonist activity was demonstrated by its ability to induce the formation of intestinal villous microfold cells in mice. This characterization of two monoclonal antibodies should now allow better evaluation of their application as therapeutic reagents and investigative tools.
\end{abstract}




\section{Introduction}

RANK (Receptor activator of NF-KB) is a signaling receptor [3] engaged by its ligand RANKL [4] but blocked by OPG (osteoprotegerin) that binds RANKL and prevents it from recognizing RANK [5] (for review see [6]). The RANK-RANKL-OPG triad was originally identified as a regulator of bone mass based on expression of the signaling receptor by pre-osteoclasts and their differentiation into mature osteoclasts by RANKL $[5,7,8]$. Thus, mice deficient for or overexpressing any of the three proteins display alterations in bone density. The triad is under the regulatory control by different factors, among which sex hormones that link osteoporosis with menopausal hormonal changes or hormone ablation therapy [9]. Hence, a treatment of osteoporosis consists of the administration of Denosumab, a human monoclonal RANKL-neutralizing antibody [10]. Similar reagents are used in animal models, such as anti-RANKL monoclonal antibody IK22-5 [2,11], RANK-Fc fusion protein $[12,13]$ or recombinant OPG [14]. A murine RANK-specific monoclonal antibody (R12-31) has been generated, which was reported as unable to block the binding of RANK-Fc to RANKL [2]. Recently, an antagonist RANK single-chain variable fragment (scFv) reactive against human and mouse RANK has been identified with a capacity to inhibit osteoclastogenesis [1].

The role of RANK is not limited to bone. Macrophages and dendritic cells are of the same lineage as osteoclasts and express this receptor. RANK activation of these cells enhances the immune response by increased cell survival and cytokine production [3,4,8,15-20]. Also epithelial cells are responsive to RANKL. Thymic medullary epithelial cells require RANK activation signals for maturation [21-23] (for review see [24]), the development of intestinal microfold epithelial cells is dependent on RANK signals $[25,26]$, and RANK activation is required for the development of lactating mammary gland epithelial cells during pregnancy [27-29] (for review see [9]). However, although studies employ RANK-specific antibodies $[13,25,30,31]$, these antibodies remain insufficiently characterized.

Therefore, in addition to potential therapeutic applications, specific antibodies are important investigative tools to study the distribution and function of RANK, RANKL and OPG. The RANKL monoclonal antibody IK22-5 [2] was used to demonstrate the production of RANKL by osteocytes [32], T cells [32-34], innate lymphoid cells [35] or lymph node stromal cells [36]. The monoclonal antibody R12-31 has shown RANK expression in synovial tissue and the myeloid cell lineage [37-39]. Although reported as non-antagonist [2] a rigorous analysis of its affinity and its biological activity against mouse and human RANK had not been published. Recently, a scFv reactive against RANK has been generated by phage display [1]. It was shown to recognize human RANK by ELISA, by immunoblot and by immunofluorescence on human osteoclasts and to inhibit osteoclastogenesis of the murine RAW cell line. A detailed affinity and functional analysis of the scFv to evaluate its utility in preclinical studies and investigative research was not performed.

In the present study, we generated an antibody (RANK-02) from the amino acid sequence of RANKbinding scFv and compared it with the anti-RANK monoclonal antibody R12-31 with regard to biological activity, interference with RANKL-RANK interaction, distinct or shared epitopes and recognition of primary RANK-expressing cells. We find that both mAbs recognize human and mouse RANK with high affinity but differ in their biological activity. Both mAbs were used to identify RANKexpressing primary cells and R12-31 was administered to mice to induce the differentiation of intestinal microfold cells. 


\section{Material and Methods}

\subsection{Production of RANK-02 antibody}

The variable domains from the anti-RANK scFv [1] were cloned into human IgG1 heavy chain and light chain expression vectors. These were co-transfected into Chinese Hamster Ovary cells, and the IgG1, named RANK-01, was purified using MabSelect SuRe (GE Healthcare). RANK-01 demonstrated severe aggregation on production, correlating with the aggregation pattern seen originally with the antiRANK scFv (see Lam M., Master's thesis, University of Alberta, 2011; https://era.library.ualberta.ca/public/view/item/uuid:0036405c-4d76-49a0-b2c7-21c148af44f4). Analysis of the variable domain sequence identified the absence of a canonical heavy chain framework methionine residue at Kabat position 82 . Site directed mutagenesis was therefore employed to insert the missing codon, generating a new human IgG heavy chain vector. This vector was co-transfected with the existing light chain vector to generate an improved anti-RANK IgG1, named RANK-02, which did not aggregate on production.

\subsection{ELISA}

For ELISA with RANK-02, recombinant human and mouse RANK-Fc were coated overnight at $20 \mu \mathrm{g} / \mathrm{ml}$ in PBS in 96 well plates (Nunc). Plates were washed with PBS, blocked for $1 \mathrm{~h}$ at room temperature with PBS containing 3\% (w/v) milk powder and washed again. RANK-02 and an isotype control lgG1 were added, the plates incubated for one hour at room temperature and washed three times with PBS/0.1\% Tween 20. Peroxidase-coupled goat anti-human kappa light chain antibody (Sigma, A7164) was diluted 1:5000, $50 \mu \mathrm{l}$ was added to each well and the plates were incubated for $1 \mathrm{~h}$. After three washes with PBS/0.1\% Tween 20, $50 \mu$ l of tetramethylbenzidine (TMB, Sigma Aldrich) was added to each well and incubated for five minutes before neutralization with $50 \mu \mathrm{l} 0.5 \mathrm{M} \mathrm{H}_{2} \mathrm{SO}_{4}$. Plates were read on a PerkinElmer Envision 2100 plate reader measuring absorbance at $450 \mathrm{~nm}$ and analyzed in GraphPad PRISM. For ELISA with R12-31, hRANK and mRANK-Fc were coated at $1 \mu \mathrm{g} / \mathrm{ml}$ in PBS for $3 \mathrm{~h}$ at $37^{\circ} \mathrm{C}$. Plates were blocked with PBS containing $4 \%(\mathrm{w} / \mathrm{v})$ milk powder and $0.05 \%$ Tween 20 for $1 \mathrm{~h}$ $37^{\circ} \mathrm{C}$. Titrated amounts of R12-31 were added, the plates incubated for $1 \mathrm{~h}$ at $37^{\circ} \mathrm{C}$ and washed 3 times with PBS/0.05 \% Tween 20. Peroxidase-coupled goat anti-rat IgG, light chain specific (Jackson Immunoreasearch) was diluted 1:5000, $100 \mu \mathrm{l}$ was added to the plates and the plates were incubated

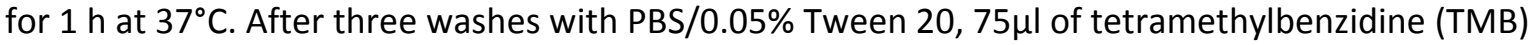
was added to each wells and then neutralized with $25 \mu \mathrm{HCL} 1 \mathrm{~N}$. Plates were read at $450 \mathrm{~nm}$ on Multiskan Ex (MTX Labsystems Inc.).

For the competitive ELISA assay, hRANK-Fc was coated at $5 \mu \mathrm{g} / \mathrm{ml}$. After blocking, wells were incubated for $1 \mathrm{~h}$ at $37^{\circ} \mathrm{C}$ with titrated amounts of the four antibodies (RANK-02 hlgG1 anti-RANK, NIP288 hlgG1 control; R12.31 rat IgG2a anti-mRANK, rat IgG2a control). Without washing, a constant volume of conditioned supernatant of 293T cells transfected with Flag-ACRP-hRANKL (5 $\mu$ l) or FlagmRANKL $(20 \mu \mathrm{l})$ was added to wells. Volumes were chosen for their abilities to generate a close to maximal but non-saturating signal. Binding of Flag-ACRP-hRANKL was revealed with biotinconjugated M2-antibody $(0.5 \mu \mathrm{g} / \mathrm{ml}$ ) (Sigma) and streptavidin-HRP (Jackson immunoresearch) (1/4000).

\subsection{Affinity measurements by surface plasmon resonance}

Real-time binding kinetics to mRANK-Fc (Sigma-Aldrich) and hRANK-Fc (purified from transfected HEK 293 cells) were measured on a BIAcore 3000. The proteins were immobilized by injecting $6 \mu \mathrm{g}$ of RANK-Fc on a CM5 chip activated with $N$-ethyl- $N$ '-dimethylaminopropyl carbodiimide/ $N$ - 
hydroxysuccinimide (GE-Healthcare, Uppsala, Sweden) in formate buffer, $\mathrm{pH} 4.3$, which gave a signal of approximately $2000 \mathrm{RU}$. Free active sites were quenched with ethanolamine hydrochloride, pH8.5. Binding was performed in $10 \mathrm{mM}$ HEPES-buffered saline containing $0.005 \% \mathrm{v} / \mathrm{v}$ surfactant P20 at 25 ${ }^{\circ} \mathrm{C}$ with a constant flow rate of $30 \mu \mathrm{l} / \mathrm{min}$. The antibodies were injected at $0.1 \mathrm{nM}$ to $4 \mathrm{nM}$ (RANK-02) and $2 \mathrm{nM}$ to $70 \mathrm{nM}$ for R12-31. The sensor chip was regenerated after each experiment by injecting $10 \mu$ of EDTA $0.5 \mathrm{M}, \mathrm{pH}$ 8. The kinetic parameters were calculated using the BIAeval 4.1 software, and the analysis used the simple Langmuir binding model $k_{\text {on }} / k_{\text {off. }}$. Specific binding profiles were obtained by subtracting the response signal from the control channel (activated/desactivated) and from blank-buffer injection. The fitting of each model was judged by the $\chi^{2}$ value and randomness of residue distribution compared to the theoretical model (Langmuir binding 1:1).

\subsection{Flow cytometry}

All labelling were performed at $4^{\circ} \mathrm{C}$ in PBS, $2 \%$ fetal calf serum (FCS), $2.5 \mathrm{mM}$ EDTA with the following antibodies: anti-RANK R12-31, anti-RANK RANK-02, rat IgG2a isotype control (BectonDickinson [BD]Pharmingen, San Jose, CA, USA), NIP228 human IgG1 isotype control (Medlmmune Inc.), donkey antirat Ig-PE (Jackson Immunoresearch), donkey anti-human Ig-PE (Jackson Immunoresearch). For flow cytometric analysis of human skin the following antibodies were used: anti-HLA DR (L203, R\&D Systems), anti-DC-SIGN (DCN46, BD-Pharmingen), anti-Langerin (929F3.01, Dendritics, Lyon, France), anti-CD14 (M5E2, BD-Pharmingen). Fixable Viability Dye eFluor ${ }^{\circledR} 780$ (ebioscience) was used to exclude the dead cells. Flow cytometry was performed on a FACS-Calibur (BD) or Gallios (BeckmanCoulter, Fullerton, CA, USA) and the flow cytometry data were analyzed using the FlowJo software (Treestar, Ashland, OR, USA). Langerhans and dermal dendritic cells were isolated from human skin as described [40].

To determine recognition of different epitopes, Jurkat JOM2 mRANK:Fas and Jurkat JOM2 hRANK:Fas were incubated on ice for 15 min with increasing amounts of unlabeled competitor antibody, followed without washing by a fixed concentration of biotinylated test mAb for $20 \mathrm{~min}$ on ice. Cells were then washed and binding of test $\mathrm{mAb}$ revealed with PE-conjugated streptavidin.

\subsection{Cell culture}

All cells were maintained in a humidified incubator with $5 \% \mathrm{CO}_{2}$ at $37^{\circ} \mathrm{C}$. Jurkat JOM2, Jurkat JOM2 mRANK:Fas and Jurkat JOM2 hRANK:Fas, were grown in RPMI 1640 medium supplemented with $10 \%$ FCS. Jurkat JOM2 mRANK:Fas and Jurkat JOM2 hRANK:Fas were generated according to a described protocol [41,42]. HEK 293 cells stably transfected with mRANK [31] or with hRANK (a kind gift of Dr. D.Heymann, University of Nantes, France) were grown in DMEM supplemented with $10 \%$ FCS, gentamicin $(10 \mu \mathrm{g} / \mathrm{ml})$ and penicillin/streptomycin $(100 \mathrm{U} / \mathrm{ml})$. G418 selection $(500 \mu \mathrm{g} / \mathrm{ml})$ was used to maintain HEK 293-mRANK cell line.

\subsubsection{Cell viability assay}

Jurkat JOM2, Jurkat JOM2 mRANK:Fas and Jurkat JOM2 hRANK:Fas $\left(50 \times 10^{3}\right.$ cells $/ 100 \mu$ l) were incubated for $16 \mathrm{~h}$ with ligands or antibodies as indicated. For RANK-02 blocking assay, $75 \times 10^{3}$ cells $/ 100 \mu$ l were incubated for $1 \mathrm{~h}$ with $50 \mu \mathrm{g} / \mathrm{ml}$ RANK-02 antibody before treatment with GSTmRANKL for $6 \mathrm{~h}$. Cell viability was then assessed using the Cell titer 96 Aqueous One Solution (Promega) according to manufacturer's instructions. Absorbance was measured at $490 \mathrm{~nm}$ using a Mithras LB940 reader (Berthold technologies). All assays were performed in duplicates and repeated at least three times. Untreated cells were set at $100 \%$ control level of cell viability. 


\subsubsection{NF- $\mathrm{KB}$ reporter gene assay}

HEK 293-mRANK and HEK 293-hRANK were transiently transfected with NF-KB1 luciferase reporter vector (Panomics) and an internal control EF1- $\beta$-galactosidase plasmid (a kind gift from Sylvie Mémet, Institut Pasteur, Paris, France) using lipofectamine 2000 (Life Technologies). $24 \mathrm{~h}$ after transfection, cells were treated in duplicates with ligands or antibodies as indicated. After $16 \mathrm{~h}$ of treatment, cells were lysed using reporter lysis buffer (Promega). Luciferase activity was measured by luminescence on a Mithras LB940 reader (Berthold Technologies) using luciferase assay system (Promega). Beta-galactosidase activity was used to normalize the luminescence values for transfection efficiency. It was measured by reading absorbance at $450 \mathrm{~nm}$ on Multiskan Ex (MTX Labsystems Inc.) using $\beta$-galactosidase enzyme assay system (Promega). To assay RANK-02 antagonist activity, transfected HEK 293-mRANK were pretreated for $1 \mathrm{~h}$ with RANK-02 at $50 \mu \mathrm{g} / \mathrm{ml}$ before adding GST-mRANKL at $0.1 \mu \mathrm{g} / \mathrm{ml}$ for $6 \mathrm{~h}$. IK22-5 anti-RANKL antibody was used at $10 \mu \mathrm{g} / \mathrm{ml}$ as a positive control for inhibition of RANK-RANKL interaction.

\subsection{Villous microfold cell induction by R12-31 and GST-mRANKL administration}

C57BL/6 mice were treated i.p. with $100 \mu \mathrm{g}$ of anti-RANK R12-31 antibody on days 0, 2, and 4 or with $50 \mu \mathrm{g}$ i.p. of GST-mRANKL on day 0 plus $100 \mu \mathrm{g}$ GST-mRANKL s.c. on days 0-4. On day 5, the proximal ileum was removed for tissue analysis. The tissue was stained with rhodamine-UEA-I as previously described [25].

\subsection{Serum TRACP $5 b$ detection after RANK-02 and GST-mRANKL administration}

Adult C57BL/6 mice were treated i.p with RANK-02 antibody $1 \mathrm{~h}$ before administration of GSTmRANKL ( $25 \mu \mathrm{g} /$ mouse) i.p. Fifteen hours afterwards, serum was taken for the measure of TRACP $5 b$ by ELISA according to the manufacturer's instructions (Immunodiagnostic systems).

\subsection{Statistical analysis}

Statistical significance was calculated by one or two way ANOVA/Bonferroni using the PRISM software. ${ }^{*}<0.05, * *<0.01, * * *<0.001, * * * *<0.0001, \mathrm{~ns}=$ not statistically significant.

\section{Results}

\subsection{Specificity and affinity of R12-31 and RANK-02 for mouse RANK}

The nucleic acid sequence obtained from a human RANK-Fc-selected scFv [1] was modified to generate a recombinant human antibody, termed RANK-02 (see Material and Methods). We first investigated its affinity and specificity for mouse $(m)$ RANK and could expect recognition of mRANK because the scFv inhibited osteoclast formation of a murine cell line. However, no further analyses were reported [1]. To first evaluate binding to mRANK we performed an ELISA in comparison with the known RANK-specific mAb R12-31 [2]. Both antibodies recognized the fusion protein in a dose dependent manner (Fig. 1A). Affinities of both mAbs were determined by surface plasmon resonance, and they bound mRANK-Fc with high affinity ( $0.2 \mathrm{nM}$ ) (Table 1). To further confirm their specificity, we performed flow cytometry analysis on two different cell lines both expressing the extracellular domain of mRANK and compared the signal intensity with that obtained with the parental cell lines. We used the Jurkat human T cell line, deficient for Fas (Jurkat JOM2) and stably expressing the mRANK extracellular domain fused to the transmembrane and intracellular domains 
of human Fas (Jurkat JOM2 mRANK:Fas) [41], and the human embryonic kidney cells HEK 293 cells, stably transfected with full-length mRANK (HEK 293-mRANK). The antibodies failed to recognize parental cell lines but bound the mRANK-expressing cells (Fig. 1B). The analysis of the mean fluorescence intensity showed that R12-31 generated a slightly stronger signal than RANK-02 (Supplementary Fig. 1A, B). Therefore, RANK-02 and R12-31 specifically recognize the extracellular domain of mRANK with high affinity.

\subsection{Biological activity of R12-31 and RANK-02 on mouse RANK}

Like any TNF receptor superfamily member, RANK signaling requires multimerization to recruit downstream signaling adaptors. The biological activity of antibodies was first characterized using the JOM2 mRANK:Fas cell line. A comparison between JOM2 mRANK:Fas cells and the parental cell line showed that GST-mRANKL specifically killed JOM2 mRANK:Fas cells by engaging the surrogate Fas apoptotic signaling pathway (Supplementary Fig.2A). Apoptosis was not observed in response to GST alone (Supplementary Fig.2B). Incubation of JOM2 mRANK:Fas cells with R12-31 led to cell death (Fig. 2A), while its isotype control (data not shown) and the RANK-02 antibody had no observed effect even at high concentrations (Fig. 2B). These results suggest that R12-31 and RANK-02 antibodies have distinct biological properties. These findings were validated using a canonical NF-KB activation assay in HEK293 cells transfected with full-length mRANK. Stimulation of these cells with GST-mRANKL as a positive control or with R12-31 both induced NF-KB activation, while RANK-02 did not (Fig. 2C, D). R12-31 and RANK-02 were used at $25 \mu \mathrm{g} / \mathrm{ml}$ and $50 \mu \mathrm{g} / \mathrm{ml}$, respectively, to be in line with the concentrations of $100 \mathrm{nM}$ of scFV used in Newa et al. and the expected low biological activity of the latter (Fig. 2B) [1]. Because the RANK-02 equivalent scFv could inhibit osteoclastogenesis [1], we next assayed the antagonist activity of RANK-02. Indeed, when Jurkat JOM2 mRANK:Fas cells were pre-incubated with $50 \mu \mathrm{g} / \mathrm{ml}$ RANK-02 prior to addition of recombinant mRANKL, cell death was almost completely inhibited (Fig. 2E). Similarly, the anti-RANK-02 antibody diminished NF-KB activation by recombinant RANKL (Fig. 2F), but again less efficiently than the antiRANKL antibody IK22-5 (Fig. 2E, F). Altogether, the above data demonstrate that R12-31 and RANK02 bind mRANK with similar affinities, but differ in their biological effects.

\subsection{Specificity and affinity of R12-31 and RANK-02 for human RANK}

We extended these analyses to human (h) RANK and observed that RANK-02 and R12-31 also recognized hRANK-Fc in ELISA (Fig. 3A). We next compared the affinity of both antibodies to hRANKFc by surface plasmon resonance and obtained an affinity of RANK- 02 for hRANK in the subnanomolar range $(0.12 \mathrm{nM})$ while R12-31 recognized hRANK with a 200-fold lower affinity (25 nM) (Table 1). To test if the antibodies recognized hRANK-transfected cell lines, we used JOM2 hRANK:Fas cells and HEK 293 cells transfected with full length hRANK. Both antibodies bound Jurkat JOM2 hRANK:Fas and HEK 293-hRANK, but not their parent cell lines (Fig. 3B). Although R12-31 labelled hRANK-expressing cells by flow cytometry, it did so less efficiently than RANK-02, as its MFI values were lower compared to those obtained with RANK-02 (Supplementary Fig. 1C, D). This may be attributable to the lower affinity of R12-31 to hRANK. This confirms that the specificity of these antibodies extends to hRANK.

\subsection{Biological effects of R12-31 and RANK-02 on human RANK}

We used cell lines expressing hRANK or hRANK:Fas fusion protein to investigate the biological effects of the antibodies on hRANK. R12-31 again displayed agonist activity (Fig. 4A) with an EC50 of 5.7 $\mathrm{ng} / \mathrm{ml}$, comparable to that observed with mRANK:Fas cells (EC50 of $2.4 \mathrm{ng} / \mathrm{ml}$ ). However, as hRANK:Fas reporter cells are about 6 times more sensitive than mRANK:Fas reporter cells 
(Supplementary Fig. 2C, D), the agonist activity of R12-31 is altogether roughly more than 10-fold less efficient on hRANK:Fas cells compared to mRANK:Fas cells, in line with its lower affinity for hRANK. RANK-02 exhibited weak agonist activity on hRANK:Fas cells at high concentrations (EC50 of $470 \mathrm{ng} / \mathrm{ml}$ ) that however never induced complete apoptosis (Fig. 4B). This apparent discrepancy with results obtained on mRANK:Fas cells might be explained by the intrinsic higher sensitivity of hRANK:Fas cells to apoptosis. Isotype controls had no effect at the highest dose tested for R12-31 and RANK-02 (Fig. 4C). We next performed the NF- $\kappa B$ reporter assay in HEK 293-hRANK cells and observed agonist effects for both mAbs with RANK-02 displaying again weaker activity (Fig. 4D). These findings confirm that R12-31 is an agonist for RANK, across the species, and revealed a weak agonist effect of RANK-02 on hRANK.

\subsection{RANK-02 efficiently interfered with RANK-RANKL interaction and recognized an} epitope distinct from R12-31

In order to understand the molecular mechanism by which RANK-02 antagonized the action of RANKL on RANK-expressing cells, we measured its ability to interfere with the binding of Flag-RANKL to FcRANK in an ELISA-based assay. Pre-incubation of coated hRANK-Fc with RANK-02 efficiently prevented further binding of human or mouse RANKL, strongly suggesting that the binding sites of RANK-02 and RANKL on hRANK overlap (Fig. 5A). In contrast, pre-incubation of coated hRANK-Fc with the mAb R12-31 did not prevent the binding of hRANKL, and only marginally interfered with the binding of mRANKL, indicating that its binding site is distinct, or only very partially common with that of hRANKL and mRANKL, respectively (Fig. 5A). Control antibodies did not interfere with RANKL-RANK interactions. To further address the question of overlapping or distinct epitopes between the mAbs, we performed a competition assay for their binding to hRANK-, or to mRANK-expressing cells by FACS. To this end, $h$ /mRANK:Fas cells were incubated with increasing concentrations of unlabeled RANK-02, R12-31, or their isotype controls, followed by biotinylated RANK-02 and phycoerythrinconjugated streptavidin. Cell labelling was determined by FACS and the MFI calculated. This was also performed for R12-31. As depicted in supplementary Fig. 3 and summarized in Fig. 5B, RANK-02 competes only with biotinylated RANK-02 on h/mRANK, and R12-31 competes only with biotinylated R12-31 on h/mRANK, showing that RANK-02 and R12-31 recognize distinct epitopes.

\subsection{Labelling of RANK on primary human Langerhans cells with RANK-02 and R12-31}

To investigate whether the antibodies are suitable for the detection of endogenous RANK, we incubated them with primary human skin dermal dendritic cells and Langerhans cells. Langerhans cells have previously been shown to express RANK using the mAb683 (R\&D Systems) $[18,19]$. Whether the related dermal dendritic cells also express RANK is not known. After allowing cells to migrate from human skin into culture medium, they were harvested and labelled for different markers to identify three subsets (Fig. 6A). The Langerhans cells (subset I) was clearly recognized by both RANK-02 and R12-31 mAbs (Fig. 6B), the CD14 dermal dendritic cells (subset II) expressed low levels of RANK, and the CD14 $4^{+}$population (subset III) was devoid of RANK (Fig. 6C, D). Both mAbs are valid tools to detect RANK expression on primary cells, as the values of \% positive Langerhans cells (Fig. 6E) and the MFI (Fig. 6F) were not significantly different. The data show that migrated human Langerhans cells express the highest levels of RANK among human skin dendritic cells.

\subsection{Assessment of antibody activity in vivo}

To assess mAb activities in vivo, we determined the capacity of RANK-02 to inhibit recombinant RANKL to activate the release of the tartrate-resistant acid phosphatase isoform $b$ (TRACP $5 b$ ) by 
osteoclasts [43,44]. Mice received increasing doses of RANK- $021 \mathrm{~h}$ before administration of $25 \mu \mathrm{g}$ GST-RANKL ( $1 \mathrm{mg} / \mathrm{kg}$ ), and TRACP $5 \mathrm{~b}$ was measured in the serum by ELISA $15 \mathrm{~h}$ later. RANKL resulted in a 30\% increase of TRACP 5b, however RANK-02 did not significantly alter this level (Fig. 7A). We had previously shown that the administration of GST-mRANKL induced the ectopic differentiation of cells of the villous small intestinal epithelium into microfold (M) cells [25]. We therefore tested whether R12-31 could induce the formation of villous $M$ cells. To this end, mice were left untreated or received either R12-31 or GST-mRANKL for 5 days. Thin strips of intestinal tissue were then prepared for staining with rhodamine UEA-I that labels M cells. Small intestinal UEA-I ${ }^{+}$villous M cells were readily observed in mice treated with R12-31 or with recombinant RANKL, in contrast to untreated mice that showed only few UEA-I cells (Fig. 7B, C). This shows that R12-31 exerts agonist activity in vivo.

\section{Discussion}

We estimated that a detailed characterization of two RANK-specific mAbs, one derived from a scFv selected against hRANK and one generated in the rat against mRANK $[1,2]$, would be beneficial to their evaluation as therapeutic reagents and investigative tools. We found that both mAbs bound to mouse and human RANK-Fc in ELISA and surface plasmon resonance and recognized RANKexpressing cell lines or primary cells in flow cytometry. The mAbs recognized distinct epitopes on RANK and displayed differences in their biological activities and in their capacity to inhibit RANKLRANK interaction. We showed that the agonist mAb R12-31 is functional in vivo.

RANK-02 and R12-31 recognized human and mouse RANK in ELISA, plasmon resonance and in flow cytometry. Cross-reactivity between RANK from both species can be expected because mouse and human RANK share 73\% sequence identity [3]. R12-31, which was generated by immunization of rats with mRANK-transfected cells, had a high affinity for mRANK but a lower affinity for hRANK. Although R12-31 comparatively stained mRANK-transfected cells better than hRANK-transfected ones, it was good enough to label human Langerhans cells. RANK-02 was obtained using a phage display selection with the hRANK extracellular domain and had a high affinity for both human and mouse RANK. Thus, this mAb may be the reagent of choice to detect human cells expressing low levels of RANK, such as CD14 dermal dendritic cells.

Biological activities of these antibodies were determined using cell lines stably expressing either RANK:Fas fusion proteins or full-length RANKs. Data showed that R12-31 was agonist on both human and mouse RANK, however, in line with its higher affinity, the activity was stronger on mRANK. A competitive ELISA further revealed that R12-31 most probably recognized a site distinct from that occupied by RANKL. This is compatible with a scenario upon which engagement of RANK by R12-31 induces conformational changes and/or aggregation of RANK that all in all mimic the action of the ligand. The biological activity of R12-31 was demonstrated in vivo by its ability to trigger the conversion of intestinal cells into M cells. The biological assays for RANK-02 yielded species-specific results: an antagonist activity was observed for mRANK and an agonist effect was seen for the human orthologue. However, its antagonist effect was weaker than that seen with anti-RANKL IK22-5 antibody, and the agonist activity may only have been detectable because of the high sensitivity of the cellular assays. In vivo, RANK-02 proved unable to inhibit recombinant RANKL-stimulated osteoclast activity. The competitive ELISA assay showed that RANK-02 competed at low concentrations with mouse and human RANKL for binding to hRANK. Thus, the capacity of RANK-02 to interfere with receptor-ligand interaction can explain its in vitro antagonist activity, while its agonist activity is likely triggered by its direct binding to RANK. EDAR is like RANK a TNF family 
member, and in a previous study characterizing a panel of anti-EDAR antibodies, it was found, using EDAR:Fas reporter cells, that several antibodies were agonist in this system while others were not. What correlated with agonist activity in this experimental system was not the affinities of antibodies, but their ability to detach slowly once bound (small $k_{\text {off }}$ [ [42]. Interestingly, RANK-02 and R12-31 have the same affinity for mRANK but R12-31 has a lower $k_{\text {off }}$ rate. Differences in agonist activities between RANK-02 and R12-31 might also reflect binding epitopes that may be more or less favorable for generating an active conformation of the receptor. Indeed, we found that the mAbs recognized distinct epitopes on RANK. RANK-02 proved to be an excellent reagent for the detection of endogenous RANK on primary human skin Langerhans cells by FACS. A staining protocol with both antibodies would provide a strong guarantee of specificity because they recognize distinct epitopes, implying that if these antibodies cross-react with other antigens, these are unlikely to be shared. We confirm using primary human skin cells that Langerhans cells express high levels of RANK, whereas CD14 dendritic cells express RANK weakly and CD14 ${ }^{+}$dendritic cells none. It has been shown that RANK expression by dendritic cells, inclusive Langerhans cells, prolongs cell survival $[4,18]$, which may also endow Langerhans cells exiting the skin to prolong interaction with T cells. Indeed, human Langerhans cells have been shown to be more potent $T$ cell activators than dermal dendritic cells [45]. Interestingly, RANK has also been implicated in the formation of regulatory T cells [12], suggesting an implication of RANK in the regulation of immunity by Langerhans cells [46-48]. Hair follicles in their growth phase are a natural source of RANKL [31], and this phase of the hair cycle is characterized by a reduced delayed-type hypersensitivity response [49], a cutaneous reaction implicating Langerhans cells. Intestinal M cells are specialized epithelial cells implicated in the transport of antigen from the lumen to underlying cells. Peyer's patches and isolated lymphoid follicles comprise numerous $M$ cells to help generate immune responses against intestinal microflora. $M$ cells are absent in RANKL or Spi-B knock-out mice entailing the failure of the immune system to respond to intestinal pathogens. Administration of recombinant RANKL has been shown to restore $M$ cells in RANKL-/- mice and leads to an increase in M cells on small intestinal villi $[25,26]$. Here we show that RANK agonist R12-31 likewise leads to ectopic M cell differentiation of villous epithelial cells, demonstrating its agonist activity in vivo and that RANK signaling is responsible for $\mathrm{M}$ cell formation.

In conclusion, we have performed a detailed characterization of two anti-RANK mAbs with respect to affinity, specificity, activity and applications. These findings should help advance our understanding of RANK-RANKL-OPG biology and pave the way for their use in vitro and in vivo.

\section{Acknowledgements}

We thank Dominique Heymann (INSERM UMR-S 957, Université de Nantes) for HEK 293 hRANK cells, Olivier Chaloin for help in surface plasmon resonance measurements, the Medlmmune Biologics Expression team for antibody expression and purification, and members of the Strasbourg laboratory for help and discussion. CGM was supported by FP7-MC-ITN 289720 "Stroma", a convention between Prestwick Chemical and Centre National pour la Recherche Scientifique, Institut National du Cancer (2012-107), Institut National du Cancer N ${ }^{\circ}$ 2012-107 and I'Agence Nationale pour la Recherche (Program "Investissements d'Avenir", ANR-10-LABX-0034 MEDALIS; ANR-11-EQPX-022). PS is supported by grants from the Swiss National Science Foundation. 


\section{References}

[1] Newa M, Lam M, Bhandari KH, Xu B, Doschak MR. Expression, characterization, and evaluation of a RANK-binding single chain fraction variable: an osteoclast targeting drug delivery strategy. Mol Pharm 2014;11:81-89.

[2] Kamijo S, Nakajima A, Ikeda K, Aoki K, Ohya K, Akiba H, et al. Amelioration of bone loss in collagen-induced arthritis by neutralizing anti-RANKL monoclonal antibody. Biochem Biophys Res Commun 2006;347:124-132.

[3] Anderson DM, Maraskovsky E, Billingsley WL, Dougall WC, Tometsko ME, Roux ER, et al. A homologue of the TNF receptor and its ligand enhance T-cell growth and dendritic-cell function. Nature 1997;390:175-179.

[4] Wong BR, Josien R, Lee SY, Sauter B, Li HL, Steinman RM, et al. TRANCE (tumor necrosis factor [TNF]-related activation-induced cytokine), a new TNF family member predominantly expressed in T cells, is a dendritic cell-specific survival factor. J Exp Med 1997;186:2075-2080.

[5] Simonet WS, Lacey DL, Dunstan CR, Kelley M, Chang MS, Luthy R, et al. Osteoprotegerin: a novel secreted protein involved in the regulation of bone density. Cell 1997;89:309-319.

[6] Walsh MC, Choi Y. Biology of the RANKL-RANK-OPG System in Immunity, Bone, and Beyond. Front Immunol 2014;5:511.

[7] Lacey DL, Timms E, Tan HL, Kelley MJ, Dunstan CR, Burgess T, et al. Osteoprotegerin ligand is a cytokine that regulates osteoclast differentiation and activation. Cell 1998;93:165-176.

[8] Yasuda H, Shima N, Nakagawa N, Yamaguchi K, Kinosaki M, Mochizuki S, et al. Osteoclast differentiation factor is a ligand for osteoprotegerin/osteoclastogenesis-inhibitory factor and is identical to TRANCE/RANKL. Proc Natl Acad Sci USA 1998;95:3597-3602.

[9] Schramek D, SigI V, Penninger JM. RANKL and RANK in sex hormone-induced breast cancer and breast cancer metastasis. Trends Endocrinol Metab 2011;22:188-194.

[10] Bridgeman MB, Pathak R. Denosumab for the reduction of bone loss in postmenopausal osteoporosis: a review. Clin Ther 2011;33:1547-1559.

[11] Lezot F, Chesneau J, Navet B, Gobin B, Amiaud J, Choi Y, et al. Skeletal consequences of RANKL-blocking antibody (IK22-5) injections during growth: Mouse strain disparities and synergic effect with zoledronic acid. Bone 2014;20:463-463.

[12] Green EA, Choi Y, Flavell RA. Pancreatic lymph node-derived CD4(+)CD25(+) Treg cells: highly potent regulators of diabetes that require TRANCE-RANK signals. Immunity 2002;16:183-191.

[13] Gonzalez-Suarez E, Jacob AP, Jones J, Miller R, Roudier-Meyer MP, Erwert R, et al. RANK ligand mediates progestin-induced mammary epithelial proliferation and carcinogenesis. Nature 2010;468:103-107.

[14] Schett G, Redlich K, Hayer S, Zwerina J, Bolon B, Dunstan C, et al. Osteoprotegerin protects against generalized bone loss in tumor necrosis factor-transgenic mice. Arthritis Rheum 2003;48:2042-2051.

[15] Fuller K, Wong B, Fox S, Choi Y, Chambers TJ. TRANCE is necessary and sufficient for osteoblast-mediated activation of bone resorption in osteoclasts. J Exp Med 1998;188:9971001.

[16] Dougall WC, Glaccum M, Charrier K, Rohrbach K, Brasel K, De Smedt T, et al. RANK is essential for osteoclast and lymph node development. Genes Dev 1999;13:2412-2424.

[17] Cremer I, Dieu-Nosjean MC, Marechal S, Dezutter-Dambuyant C, Goddard S, Adams D, et al. Long-lived immature dendritic cells mediated by TRANCE-RANK interaction. Blood 2002;100:3646-3655.

[18] Barbaroux JB, Beleut M, Brisken C, Mueller CG, Groves R. Epidermal receptor activator of NFkB ligand controls Langerhans cell numbers and proliferation. J Immunol 2008;181:11031108.

[19] Schöppl A, Botta A, Prior M, Akgun J, Schuster C, Elbe-Burger A. Langerhans cell precursors acquire RANK/CD265 in prenatal human skin. Acta Histochem 2015;117:425-530. 
[20] Josien R, Wong BR, Li HL, Steinman RM, Choi Y. TRANCE, a TNF family member, is differentially expressed on T cell subsets and induces cytokine production in dendritic cells. J Immunol 1999;162:2562-2568.

[21] Rossi SW, Kim MY, Leibbrandt A, Parnell SM, Jenkinson WE, Glanville SH, et al. RANK signals from CD4+3- inducer cells regulate development of Aire-expressing epithelial cells in the thymic medulla. J Exp Med 2007;204:1267-1272.

[22] Akiyama T, Shimo Y, Yanai H, Qin J, Ohshima D, Maruyama Y, et al. The tumor necrosis factor family receptors RANK and CD40 cooperatively establish the thymic medullary microenvironment and self-tolerance. Immunity 2008;29:423-437.

[23] Hikosaka Y, Nitta T, Ohigashi I, Yano K, Ishimaru N, Hayashi Y, et al. The cytokine RANKL produced by positively selected thymocytes fosters medullary thymic epithelial cells that express autoimmune regulator. Immunity 2008;29:438-450.

[24] Akiyama T, Shinzawa M, Akiyama N. TNF receptor family signaling in the development and functions of medullary thymic epithelial cells. Front Immunol 2012;3:278.

[25] Knoop KA, Kumar N, Butler BR, Sakthivel SK, Taylor RT, Nochi T, et al. RANKL is necessary and sufficient to initiate development of antigen-sampling $M$ cells in the intestinal epithelium. J Immunol 2009;183:5738-5747.

[26] Kanaya T, Hase K, Takahashi D, Fukuda S, Hoshino K, Sasaki I, et al. The Ets transcription factor Spi-B is essential for the differentiation of intestinal microfold cells. Nat Immunol 2012;13:729-736.

[27] Srivastava S, Matsuda M, Hou Z, Bailey JP, Kitazawa R, Herbst MP, et al. Receptor activator of NF-kappaB ligand induction via Jak2 and Stat5a in mammary epithelial cells. J Biol Chem 2003;278:46171-46178.

[28] Fata JE, Kong YY, Li J, Sasaki T, Irie-Sasaki J, Moorehead RA, et al. The osteoclast differentiation factor osteoprotegerin-ligand is essential for mammary gland development. Cell 2000;103:41-50.

[29] Kim NS, Kim HT, Kwon MC, Choi SW, Kim YY, Yoon KJ, et al. Survival and differentiation of mammary epithelial cells in mammary gland development require nuclear retention of Id 2 due to RANK signaling. Mol Cell Biol 2011;31:4775-4788.

[30] Gonzalez-Suarez E, Branstetter D, Armstrong A, Dinh H, Blumberg H, Dougall WC. RANK overexpression in transgenic mice with mouse mammary tumor virus promoter-controlled RANK increases proliferation and impairs alveolar differentiation in the mammary epithelia and disrupts lumen formation in cultured epithelial acini. Mol Cell Biol 2007;27:1442-1454.

[31] Duheron V, Hess E, Duval M, Decossas M, Castaneda B, Klöpper JE, et al. Receptor Activator of NF-kB (RANK) stimulates the proliferation of epithelial cells of the epidermo-pilosebaceous unit. Proc Natl Acad Sci USA 2011;108:5342-5347.

[32] Nakashima T, Hayashi M, Fukunaga T, Kurata K, Oh-Hora M, Feng JQ, et al. Evidence for osteocyte regulation of bone homeostasis through RANKL expression. Nat Med 2011;17:1231-1234.

[33] Fionda C, Nappi F, Piccoli M, Frati L, Santoni A, Cippitelli M. 15-deoxy-Delta12,14prostaglandin $\mathrm{J} 2$ negatively regulates rankl gene expression in activated $\mathrm{T}$ lymphocytes: role of NF-kappaB and early growth response transcription factors. J Immunol 2007;178:40394050.

[34] Totsuka T, Kanai T, Nemoto $Y$, Tomita T, Okamoto R, Tsuchiya $K$, et al. RANK-RANKL signaling pathway is critically involved in the function of CD4+CD25+ regulatory T cells in chronic colitis. J Immunol 2009;182:6079-6087.

[35] Schmutz S, Bosco N, Chappaz S, Boyman O, Acha-Orbea H, Ceredig R, et al. Cutting edge: IL-7 regulates the peripheral pool of adult ROR gamma+ lymphoid tissue inducer cells. J Immunol 2009;183:2217-2221.

[36] Cupedo T, Vondenhoff MF, Heeregrave EJ, De Weerd AE, Jansen W, Jackson DG, et al. Presumptive lymph node organizers are differentially represented in developing mesenteric and peripheral nodes. J Immunol 2004;173:2968-2975. 
[37] Hoshino A, limura T, Ueha S, Hanada S, Maruoka Y, Mayahara M, et al. Deficiency of chemokine receptor CCR1 causes osteopenia due to impaired functions of osteoclasts and osteoblasts. J Biol Chem 2010;285:28826-28837.

[38] Arizon M, Nudel I, Segev H, Mizraji G, Elnekave M, Furmanov K, et al. Langerhans cells downregulate inflammation-driven alveolar bone loss. Proc Natl Acad Sci USA 2012;109:70437048.

[39] Hakozaki A, Yoda M, Tohmonda T, Furukawa M, Hikata T, Uchikawa S, et al. Receptor activator of NF-kappaB (RANK) ligand induces ectodomain shedding of RANK in murine RAW264.7 macrophages. J Immunol 2010;184:2442-2448.

[40] Schaeffer E, Flacher V, Papageorgiou V, Decossas M, Fauny J, Krämer M, et al. Dermal CD14+ Dendritic Cell and Macrophage Infection By Dengue Virus is Stimulated By Interleukin-4. J Invest Dermatol 2015;135:1743-1751.

[41] Schneider P, Willen L, Smulski CR. Tools and techniques to study ligand-receptor interactions and receptor activation by TNF superfamily members. Methods Enzymol 2014;545:103-125.

[42] Kowalczyk-Quintas C, Willen L, Dang AT, Sarrasin H, Tardivel A, Hermes K, et al. Generation and characterization of function-blocking anti-ectodysplasin $A$ (EDA) monoclonal antibodies that induce ectodermal dysplasia. J Biol Chem 2014;289:4273-4285.

[43] Janckila AJ, Takahashi K, Sun SZ, Yam LT. Tartrate-resistant acid phosphatase isoform $5 \mathrm{~b}$ as serum marker for osteoclastic activity. Clin Chem 2001;47:74-80.

[44] Tomimori Y, Mori K, Koide M, Nakamichi Y, Ninomiya T, Udagawa N, et al. Evaluation of pharmaceuticals with a novel 50-hour animal model of bone loss. J Bone Miner Res 2009;24:1194-1205. doi: 1110.1359/jbmr.090217.

[45] Furio L, Briotet I, Journeaux A, Billard H, Peguet-Navarro J. Human langerhans cells are more efficient than CD14(-)CD1c(+) dermal dendritic cells at priming naive CD4(+) T cells. J Invest Dermatol 2010;130:1345-1354.

[46] Loser K, Mehling A, Loeser S, Apelt J, Kuhn A, Grabbe S, et al. Epidermal RANKL controls regulatory T-cell numbers via activation of dendritic cells. Nat Med 2006;12:1372-1379.

[47] Gomez de Aguero M, Vocanson M, Hacini-Rachinel F, Taillardet M, Sparwasser T, Kissenpfennig $A$, et al. Langerhans cells protect from allergic contact dermatitis in mice by tolerizing CD8(+) T cells and activating Foxp3(+) regulatory T cells. J Clin Invest 2012;122:1700-1711.

[48] Flacher V, Tripp CH, Mairhofer DG, Steinman RM, Stoitzner P, Idoyaga J, et al. Murine Langerin+ dermal dendritic cells prime CD8+ T cells while Langerhans cells induce crosstolerance. EMBO Mol Med 2014;6:1191-1204.

[49] Hofmann U, Tokura $Y$, Ruckert R, Paus R. The anagen hair cycle induces systemic immunosuppression of contact hypersensitivity in mice. Cell Immunol 1998;184:65-73.

\section{Figure legends}

Figure 1. RANK-directed antibodies RANK-02 and R12-31 bind to the mouse RANK extracellular domain. (A) Recognition of mouse RANK-Fc fusion protein by ELISA. Data is expressed as color production (absorbance at $450 \mathrm{~nm}$ ) by enzyme-coupled secondary antibody recognizing increasing concentrations of RANK-02 and R12-31 bound to fixed concentration of surface-attached mRANK-Fc. (B) Detection by flow cytometry of mouse RANK stably expressed either by Jurkat JOM2 cells (as fusion protein between the RANK extracellular domain and the intracellular domain of human Fas) or by HEK 293 cells (as full-length RANK). Primary antibodies were either RANK-02 or R12-31 and secondary antibodies were donkey anti-human immunoglobulin or donkey anti-rat immunoglobulin, respectively, conjugated to phycoerythrin. Controls were untransfected cells and primary antibody isotypes. Data is representative of 5 independent experiments. 
Figure 2. The anti-RANK antibodies have different biological activities on mouse RANK. (A) Doseresponse curve of R12-31 antibody on Jurkat JOM2 cells expressing or not mRANK:Fas. The graph displays \% cell viability relative to Jurkat JOM2 cells grown in the absence of antibody (non-treated, $N T)$ as a function of increasing concentrations of R12-31. Data points are the mean ( $\pm S D, n=3$ with duplicate wells). Curve fitting was performed using the PRISM software. (B) As for panel A, but using the antibody RANK-02. (C) NF-KB activation by R12-31 of HEK 293 cells stably expressing full-length murine RANK and transfected with an NF-KB responsive luciferase reporter gene and a $\beta$ galactosidase expressing transfection control plasmid. Data are the mean \% relative to RANKLactivated cells ( \pm SD, $n=3$ with duplicate wells). Data are for $0.1 \mu \mathrm{g} / \mathrm{ml}$ RANKL and $25 \mu \mathrm{g} / \mathrm{ml} \mathrm{R} 12-31$ antibody. NT= non-treated cells. (D) As for panel B, but using RANK-02 antibody $(50 \mu \mathrm{g} / \mathrm{ml})$. (E) The antagonist activity of RANK-02 was tested by its capacity to block mRANK:Fas-mediated cell apoptosis triggered by recombinant GST-mRANKL. The graph presents JOM2 mRANK:Fas cell viability in the absence or presence of recombinant RANKL, the RANKL-blocking antibody IK22-5 and RANK02 , relative to non-treated cells (NT). RANKL was used at $1 \mathrm{ng} / \mathrm{ml}, \mathrm{IK} 22-5$ at $10 \mu \mathrm{g} / \mathrm{ml}$ and RANK-02 at $50 \mu \mathrm{g} / \mathrm{ml}$. (F) The antagonist effect of RANK-02 was tested by its capacity to block NF-KB activation elicited by recombinant RANKL. Data are expressed as the mean $\%$ ( $\pm S D, n=3$ with duplicate wells) relative to RANKL-induced NF- $\kappa B$ activation. RANKL was used at $0.1 \mu \mathrm{g} / \mathrm{ml}, I K 22-5$ at $10 \mu \mathrm{g} / \mathrm{ml}$ and RANK-02 at $50 \mu \mathrm{g} / \mathrm{ml}$. For data panels C-F, statistical significance was calculated by one way Anova/Bonferroni using the PRISM software.

Figure 3. RANK-directed antibodies RANK-02 and R12-31 bind to the human RANK extracellular domain. (A) Recognition of human RANK-Fc fusion protein by ELISA. Data is expressed as color production (absorbance at $450 \mathrm{~nm}$ ) by enzyme-coupled secondary antibody recognizing increasing concentrations of RANK-02 and R12-31 bound to fixed concentration of surface-attached RANK-Fc. (B) Detection by flow cytometry of human RANK stably expressed either by Jurkat JOM2 cells (as a fusion protein between the RANK extracellular domain and the intracellular domain of human Fas) or by HEK 293 cells (as full-length RANK). Detection was performed as described in Fig. 1B. Data is representative of 5 independent experiments.

Figure 4. Both antibodies are agonists on human RANK. (A) Dose-response curve of the R12-31 antibody on Jurkat JOM2 cells expressing or not hRANK:Fas. The graph displays cell viability relative to the percentage of Jurkat JOM2 cells grown in the absence of antibody as a function of increasing concentrations of anti-RANK antibody R12-31. Data points are the mean $( \pm S D, n=3$ with duplicate wells). Curve fitting was performed using the PRISM software. NT= non-treated cells. (B) As for panel A, but using the RANK-02 antibody. (C) The JOM2 hRANK:Fas cell viability assay shows that isotype controls for R12-31 (rat IgG2a, $1 \mu \mathrm{g} / \mathrm{ml}$ ) and for RANK-02 (human IgG1, $50 \mu \mathrm{g} / \mathrm{ml}$ ) have no human RANK-stimulating effect. (D) NF-KB activation by R12-31 and RANK-02 antibodies in HEK 293 cells stably expressing full-length human RANK and transfected with an NF-KB-responsive luciferase reporter gene. Data are the mean \% relative to RANKL-activated cells ( $\pm S D, n=3$ with duplicate wells). Data is shown for $0.1 \mu \mathrm{g} / \mathrm{ml}$ RANKL, $25 \mu \mathrm{g} / \mathrm{ml} \mathrm{R} 12-31$ and $50 \mu \mathrm{g} / \mathrm{ml}$ RANK-02. NT= non-treated cells. Statistical significance was calculated by one way Anova/Bonferroni using the PRISM software.

Figure 5. Analysis of shared or distinct epitopes. (A) RANKL blocking by the MAbs. ELISA plates were coated with human RANK-Fc, incubated first with the mAbs and then with human (left) or mouse (right) RANKL. The amount of bound RANKL was measured. The data show that the binding sites of RANK-02 and RANKL overlap, but that they are distinct for R12-31 and RANKL. (B) Competitive FACS to determine RANK-02 / R12-31 distinct or shared epitopes. Jurkat JOM2 mRANK:Fas and Jurkat JOM2 hRANK:Fas were incubated on ice for 15 min with increasing amounts of unlabeled competitor antibody, followed without washing by a fixed concentration of biotinylated test $\mathrm{mAb}$ for $20 \mathrm{~min}$ on 
ice. Cells were then washed and the binding of test mAb revealed with PE-conjugated streptavidin. Graphs show that RANK-02 and R12-31 do not cross-compete for binding to RANK-expressing cells.

Figure 6. The anti-RANK mAbs recognize RANK on primary human Langerhans cells. (A) Flow cytometry gating strategy to obtain three cell populations: Langerin ${ }^{+}$cells (population I), and CD14 and $\mathrm{CD} 14^{+}$dermal dendritic cells (populations II and III, respectively). Dead cells are excluded using the FVD stain. Human RANK expression by Langerhans cells (B), CD14 dendritic cells (C) and CD14 ${ }^{+}$ dendritic cells using both anti-RANK mAbs and their isotype controls (in grey). (D). The percentage of positive cells is indicated. (E) Mean percentage of RANK-positive cells \pm SD measured with RANK-02 or R12-31 staining in three different populations of dendritic cells isolated from three donors $(n=3$ donors). (F) mean of $\triangle M F I \pm S D$ measured with RANK-02 or R12-31 staining in the three different populations of dendritic cells isolated from three donors $(n=3)$. $\triangle M F I=$ MFI RANK antibody - MFI isotype control. Statistical significance was calculated by two way Anova/Bonferroni using the PRISM software.

Figure 7. Assessment of biological activities in vivo. (A) RANK-02 antagonist activity was measured as inhibition of osteoclast-dependent release of TRACP $5 b$ into serum. The data is the mean (SEM, $\mathrm{n}=4$ ) of TRACP $5 \mathrm{~b}$ in mice mock treated (PBS), or injected with $25 \mu \mathrm{g}$ GST-mRANKL alone or in the presence of increasing amounts of RANK-02. (B, C) R12-31 agonist activity was determined as the capacity to induce $M$ cells in the proximal ileum of $\mathrm{C} 57 \mathrm{BL} / 6$ mice. (B) Whole mount staining for UEA$\mathrm{I}^{+} \mathrm{M}$ cells in the proximal ileum, untreated or treated with GST-mRANKL or R12-31 mAb for 5 days. Nuclear counterstain was with DAPI. In untreated mice no villous M cells are found, whilst anti-RANK R12-31 mAb and recombinant-mRANKL induce a large number of polygonal UEA-I ${ }^{+}$villous $M$ cells. Scale bar is $100 \mu \mathrm{m}$. (C) The graph presents the cell counts of UEA-I ${ }^{+} \mathrm{M}$ cells per villus of 5 representable villous tips on the whole mount images in the three different conditions. Data is the mean $\pm S D$ of five representative villous tips for each group. Statistical significance was calculated by two way Anova/Bonferroni using the PRISM software. 
Table 1. Association rates $\left(\mathrm{K}_{\text {on }}\right)$, dissociation rates $\left(\mathrm{K}_{\text {off }}\right)$ and binding affinities $(\mathrm{KD})$ of anti-RANK antibodies on murine and human RANK-Fc were measured by surface plasmon resonance.

\begin{tabular}{|c|c|c|c|}
\hline & $K_{\text {on }}\left(M^{-1} s^{-1}\right)$ & $K_{\text {off }}\left(s^{-1}\right)$ & KD (M) \\
\hline & \multicolumn{3}{|c|}{ Mouse RANK-Fc } \\
\hline RANK-02 & $7.08 \times 10^{5}$ & $1.44 \times 10^{-4}$ & $2.03 \times 10^{-10}$ \\
\hline \multirow[t]{2}{*}{ R12-31 } & $5.14 \times 10^{4}$ & $8.99 \times 10^{-6}$ & $1.75 \times 10^{-10}$ \\
\hline & \multicolumn{3}{|c|}{ Human RANK-Fc } \\
\hline RANK-02 & $3.32 \times 10^{5}$ & $4.12 \times 10^{-5}$ & $1.24 \times 10^{-10}$ \\
\hline R12-31 & $2.32 \times 10^{3}$ & $5.97 \times 10^{-5}$ & $2.57 \times 10^{-8}$ \\
\hline
\end{tabular}



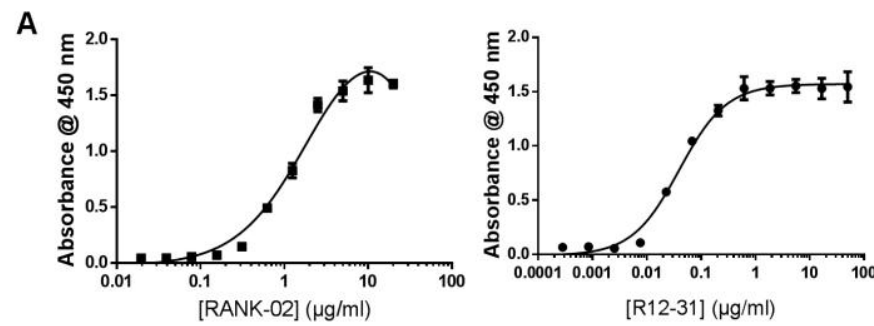

B

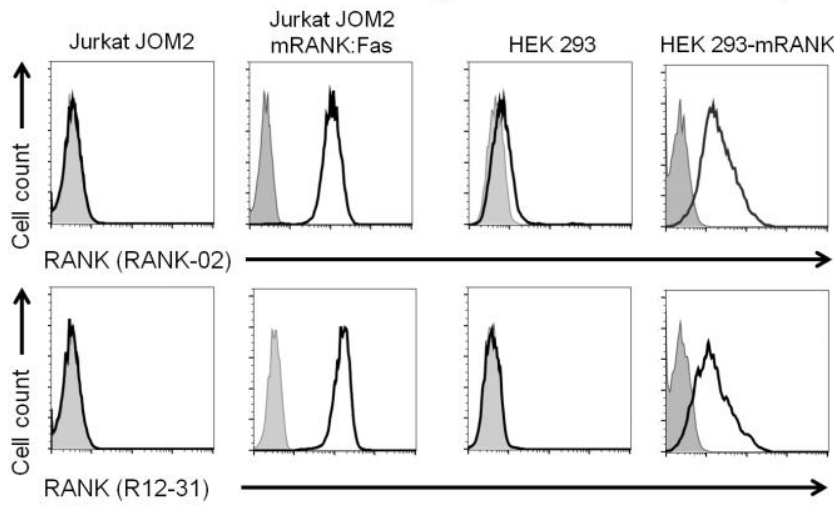

Fiqure 1 
A

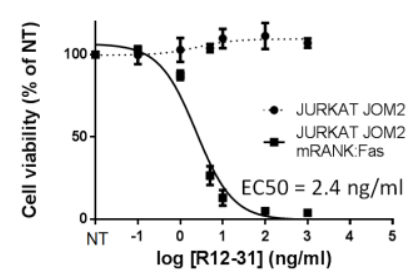

C

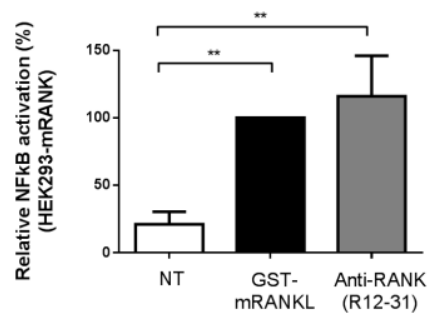

E

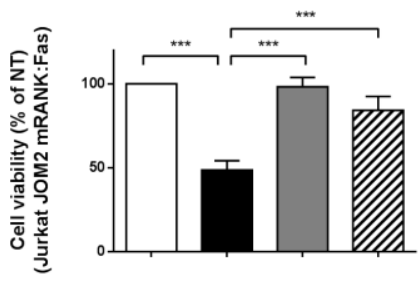

GST-mRANKL

Anti-RANKL(IK22-5)

Anti-RANK (RANK-02)
B

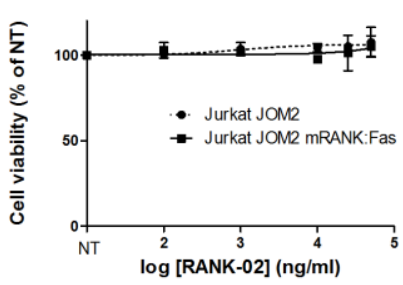

D

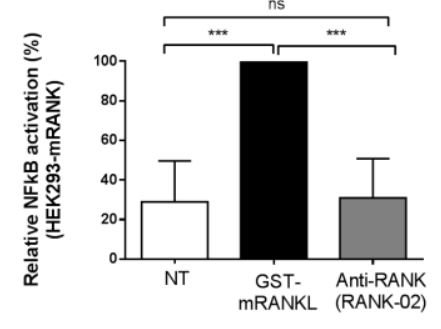

F

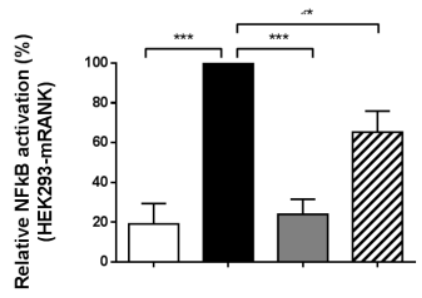

GST-mRANKL Anti-RANK (RANK-02)

\section{Figure 2}



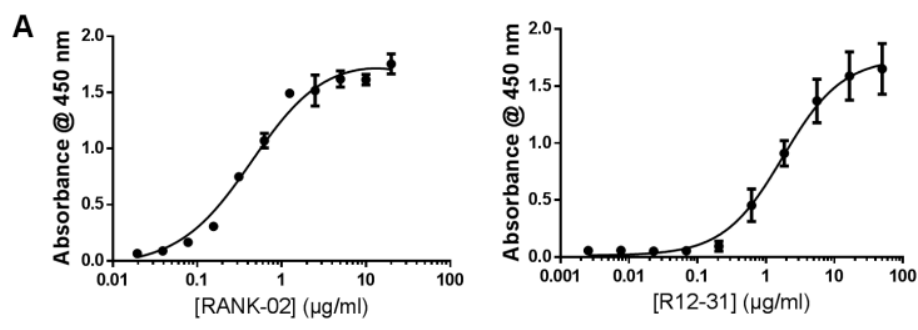

B

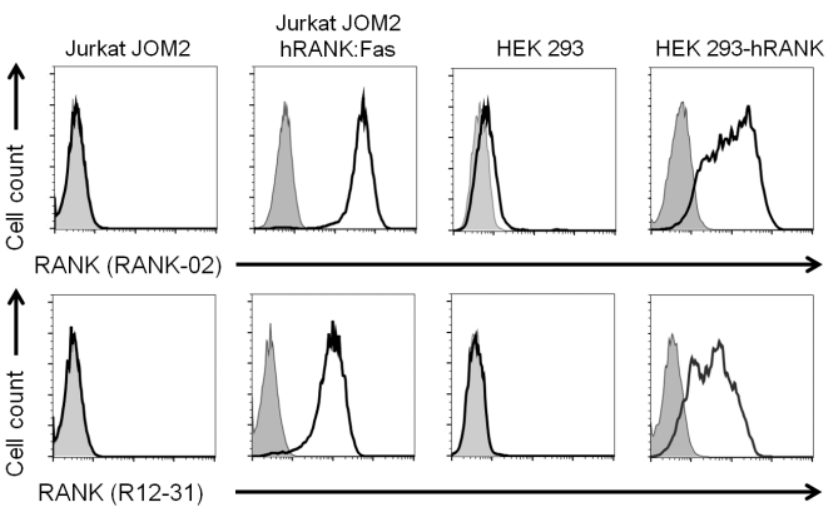

Figure 3 
A

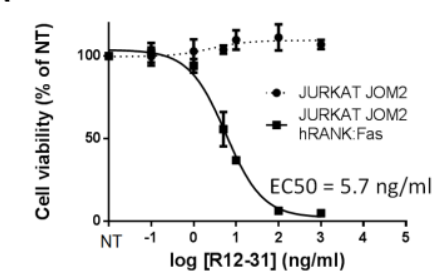

C

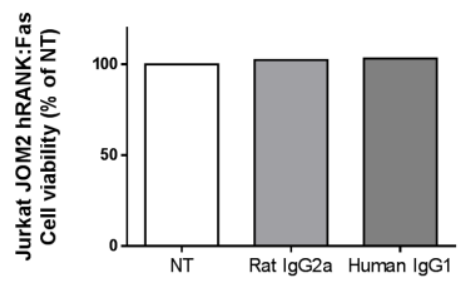

B

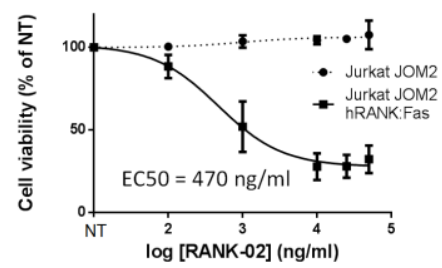

D

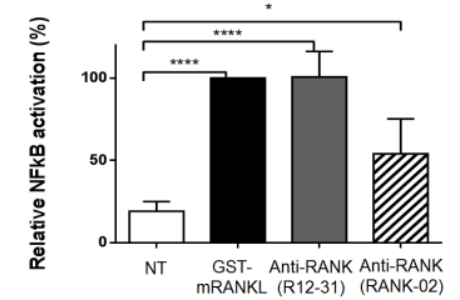

Figure 4 


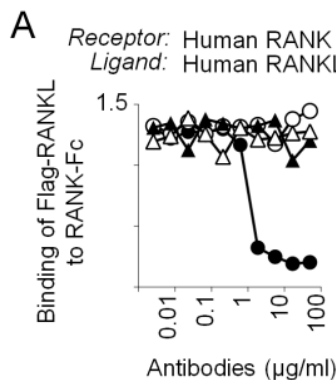

Human RANK Mouse RANKL

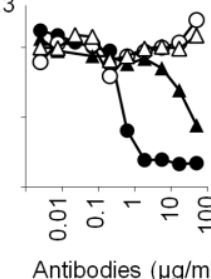

B

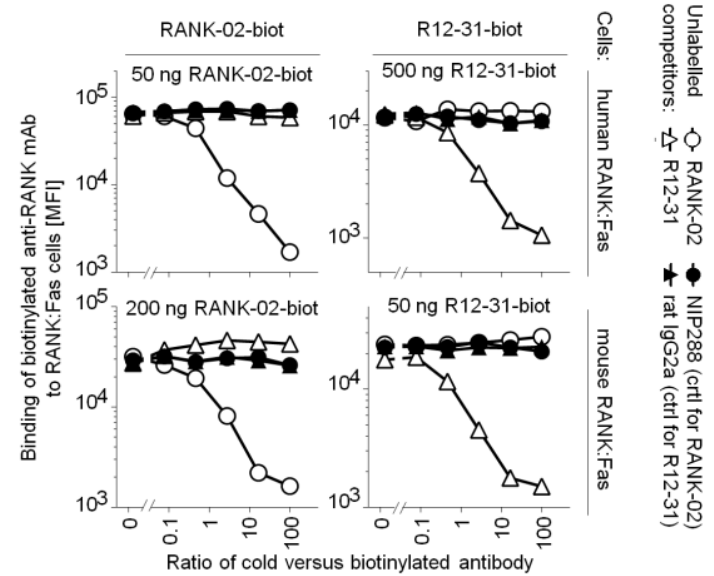

\section{Figure 5}


A

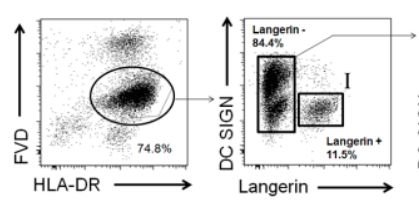

D III. CD14+ dendritic cells

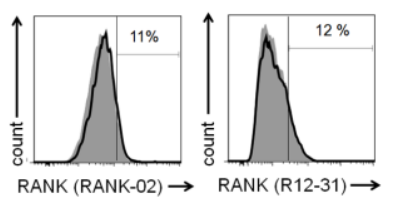

E
B I. Langerhans cells
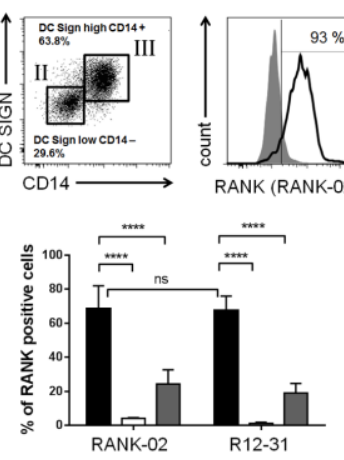

F
C II. CD14-dendritic cells
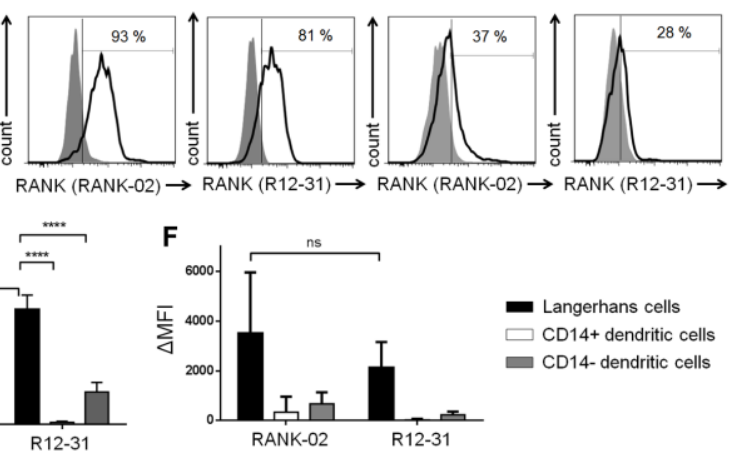

Figure 6 

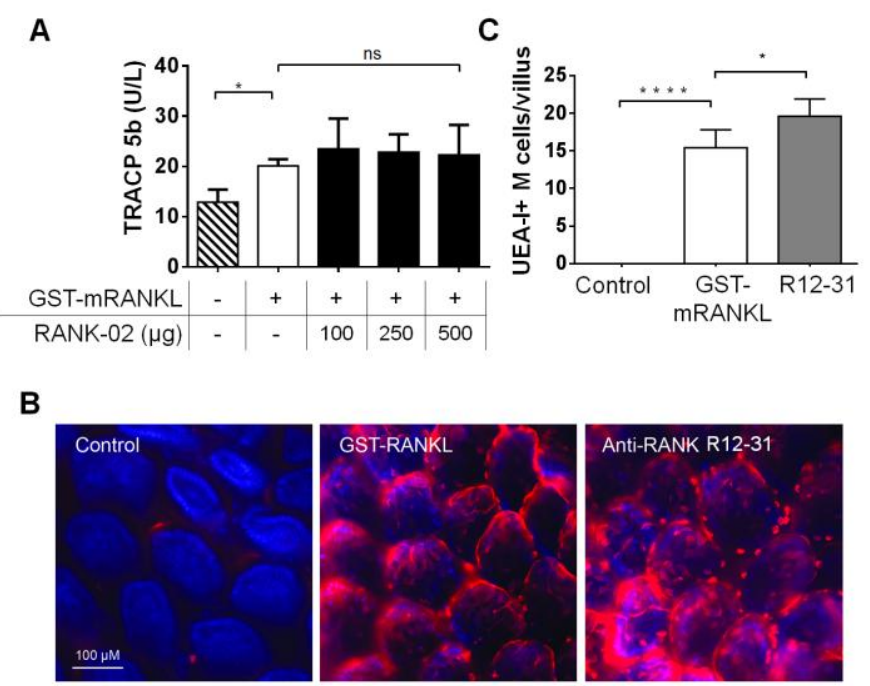

Figure 7 